\title{
Exact Solutions for a Generalized KdV-MKdV Equation with Variable Coefficients
}

\author{
Bo Tang, ${ }^{1,2}$ Xuemin Wang, ${ }^{3}$ Yingzhe Fan, ${ }^{4}$ and Junfeng $Q u^{1}$ \\ ${ }^{1}$ School of Mathematics and Computer Science, Hubei University of Arts and Science, Xiangyang, Hubei 441053, China \\ ${ }^{2}$ School of Mathematics and Statistics, Xian Jiaotong University, Xian 710049, China \\ ${ }^{3}$ Texas University at Dallas, Dallas, TX 75080-3021, USA \\ ${ }^{4}$ School of Mathematics and Statistics, Wuhan University, Wuhan, Hubei 430072, China \\ Correspondence should be addressed to Bo Tang; tangbo0809@163.com
}

Received 24 September 2015; Revised 19 December 2015; Accepted 27 December 2015

Academic Editor: Hassan Askari

Copyright (c) 2016 Bo Tang et al. This is an open access article distributed under the Creative Commons Attribution License, which permits unrestricted use, distribution, and reproduction in any medium, provided the original work is properly cited.

By using solutions of an ordinary differential equation, an auxiliary equation method is described to seek exact solutions of variablecoefficient KdV-MKdV equation. As a result, more new exact nontravelling solutions, which include soliton solutions, combined soliton solutions, triangular periodic solutions, Jacobi elliptic function solutions, and combined Jacobi elliptic function solutions, for the KdV-MKdV equation are obtained. It is shown that the considered method provides a very effective, convenient, and powerful mathematical tool for solving many other nonlinear partial differential equations with variable coefficients in mathematical physics.

\section{Introduction}

In the nonlinear science, many important phenomena in various fields can be described by the nonlinear partial differential equations (NPDEs). Searching for exact soliton solutions of NPDEs plays an important and significant role in the study on the dynamics of those phenomena. Various methods have been used to handle nonlinear partial differential equations, such as the Hirota bilinear method [1], inverse scattering method $[2,3]$ the Bäcklund transformation method [4], subequation method [5-7], F-expansion method [8-10], sine-cosine method [11,12], sech-tanh method [13,14], Exp-function method $[15,16]$, and Jacobi elliptic function method [17-19].

It is well known that NPDEs with variable coefficients are more realistic in various physical situations than their constant coefficients counterparts. However, most of the above methods are related to the constant-coefficient NPDEs. The present work is motivated by the desire to establish an auxiliary equation method to construct new and more general exact solutions of variable-coefficient NPDEs, such as soliton and soliton-like solutions, triangular periodic solutions, Jacobi elliptic function solutions, and many exact explicit solutions in form of hyperbolic function solutions and trigonometric function solutions. Being concise and straightforward, this method is applied to the generalized variable-coefficient $\mathrm{KdV}-\mathrm{MKdV}$ equation as the following form [20]:

$$
\begin{aligned}
u_{t}- & 6 f_{0}(t) u u_{x}-6 f_{1}(t) u^{2} u_{x}+f_{2}(t) u_{x x x}-f_{3}(t) u_{x} \\
& +f_{4}(t)\left(A u+x u_{x}\right)=0,
\end{aligned}
$$

where $f_{0}(t), f_{1}(t), f_{2}(t), f_{3}(t)$, and $f_{4}(t)$ are arbitrary functions of $t$.

Equation (1) can be used to describe the propagation of weakly nonlinear long waves in a KdV-typed medium by changing the coefficients of dispersion and nonlinear coefficients. It includes the following three important equations:

(a) Setting $f_{0}(t)$, (1) can be degenerated to the following variable-coefficient and nonisospectral $\mathrm{KdV}$ equation studied in $[21,22]$ :

$$
\begin{aligned}
u_{t}= & K_{0}(t)\left(u_{x x x}+6 u u_{x}\right)+4 K_{1}(t) u_{x} \\
& -h(t)\left(2 u+x u_{x}\right),
\end{aligned}
$$

which models many important nonlinear phenomena, including shallow water waves, dust acoustic solitary structures 
in magnetized dusty plasmas, and ion acoustic waves in plasmas.

(b) Setting $f_{0}(t)=0, f_{1}(t)=f_{2}(t)=-h_{1}(t), f_{3}(t)=$ $4 h_{2}(t), f_{4}(t)=h_{0}(t), A=1,(1)$ can be degenerated to the following variable-coefficient $\mathrm{MKdV}$ equation studied in [23]:

$$
\begin{aligned}
u_{t}= & h_{1}(t)\left(u_{x x x}-6 u^{2} u_{x}\right)+4 h_{2}(t) u_{x} \\
& -h_{0}(t)\left(u+x u_{x}\right),
\end{aligned}
$$

which models many important nonlinear phenomena, including shallow water waves, dust acoustic solitary structures in magnetized dusty plasmas, and ion acoustic waves in plasmas.

(c) Setting $f_{0}(t)=-a(t) / 6, f_{1}(t)=-b(t) / 6, f_{2}(t)=h(t)$, $f_{3}(t)=-d(t), f_{4}(t)=f(t) / A, x=0$, (1) becomes the following variable-coefficient Gardner equation:

$$
\begin{gathered}
u_{t}+a(t) u u_{x}+b(t) u^{2} u_{x}+d(t) u_{x}+f(t) u \\
+h(t) u_{x x x}=0,
\end{gathered}
$$

which is widely used in various branches of physics, such as plasma physics, fluid physics, and quantum field theory [24, 25]. It also describes a variety of wave phenomena in plasma and solid state.

The rest of this paper is organized as follows: in Section 2, we will describe the auxiliary equation method for finding out solutions of variable-coefficient NPDEs and give the main steps of the method here. In Section 3, we illustrate the method in detail with the generalized variable-coefficient $\mathrm{KdV}-\mathrm{MKdV}$ equation. In Section 4, some conclusions are given.

\section{Description of the Auxiliary Equation Method}

Consider a given variable-coefficient nonlinear partial differential equation with independent variable $X=\left(x_{0}=\right.$ $\left.t, x_{1}, x_{2}, \ldots, x_{n}\right)$ and dependent variable $u$ :

$$
P\left(u, u_{t}, u_{x_{i}}, u_{x_{i} x_{j}}, \ldots\right)=0,
$$

where $P$ is in general a polynomial function of its argument and the subscripts denote the partial derivatives.

Suppose (5) has the following solution:

$$
u(t, x)=\sum_{i=0}^{l} a_{i} F^{i}(\xi)
$$

where $a_{i}=a_{i}\left(x_{1}, x_{2}, \ldots, x_{n}, t\right)$ and $\xi=\xi\left(x_{1}, x_{2}, \ldots, x_{n}, t\right)$ are functions to be determined later and $F^{\prime}(\xi)=d F / d \xi$ satisfies

$$
F^{\prime 2}(\xi)=\sum_{j=0}^{m} h_{i} F^{j}(\xi),
$$

where the values of $m$ can be determined by balancing the highest differential term with the nonlinear terms in (5). The main steps by which we get exact solutions to variablecoefficient NPDE are outlined as follows.
TABLE 1: Solutions of Case 2.1. $m(0<m<1)$ denotes the modulus of the Jacobi elliptic function, $k_{1}=\sqrt{1-m^{2}}$, and $A, B, C(A B C \neq 0)$, and $D$ are arbitrary constants.

\begin{tabular}{lccc}
$h_{0}$ & $h_{2}$ & $h_{4}$ & $F(\xi)$ \\
0 & $>0$ & $<0$ & $\sqrt{-\frac{h_{2}}{h_{4}}} \operatorname{sech}\left(\sqrt{h_{2}} \xi\right)$ \\
\hline 0 & $>0$ & $>0$ & $\sqrt{\frac{h_{2}}{h_{4}}} \operatorname{csch}\left(\sqrt{h_{2}} \xi\right)$ \\
\hline$\frac{h_{2}^{2}}{4 h_{4}}$ & $<0$ & $>0$ & $\sqrt{-\frac{h_{2}}{2 h_{4}} \tanh \left(\frac{\sqrt{-h_{2}} \xi}{2}\right)}$ \\
\hline 0 & $<0$ & $>0$ & $\sqrt{-\frac{h_{2}}{h_{4}}} \sec \left(\sqrt{-h_{2}} \xi\right) \cdot \sqrt{-\frac{h_{2}}{h_{4}}} \csc \left(\sqrt{-h_{2}} \xi\right)$ \\
\hline $\begin{array}{lcc}h_{2}^{2} \\
4 h_{4}\end{array}$ & $>0$ & $>0$ & $\sqrt{\frac{h_{2}}{2 h_{4}}} \tan \left(\frac{\sqrt{h_{2}} \xi}{2}\right)$ \\
\hline 0 & 0 & $>0$ & $-\frac{1}{\sqrt{h_{4}} \xi}$ \\
\hline 1 & $-\left(1+m^{2}\right)$ & $m^{2}$ & $\operatorname{sn} \xi, \operatorname{cd} \xi=\frac{\operatorname{cn} \xi}{\operatorname{dn} \xi}$ \\
\hline
\end{tabular}

$\underline{\left(1-m^{2}\right) 2 m^{2}-1 \quad-m^{2} \quad \mathrm{cn} \xi}$

$\begin{array}{llll}m^{2}-1 & 2-m^{2} & -1 & \mathrm{dn} \xi\end{array}$

$m^{2} \quad-\left(1+m^{2}\right) \quad 1 \quad \mathrm{~ns} \xi=\frac{1}{\mathrm{sn} \xi}, \mathrm{dc} \xi=\frac{\mathrm{dn} \xi}{\mathrm{cn} \xi}$

$-m^{2} \quad 2 m^{2}-1 \quad 1-m^{2} \quad \mathrm{nc} \xi=\frac{1}{\mathrm{cn} \xi}$

$\begin{array}{llll}-1 & 2-m^{2} & m^{2}-1 & \text { nd } \xi=\frac{1}{\operatorname{dn} \xi}\end{array}$

$1 \quad 2-m^{2} \quad 1-m^{2} \quad \mathrm{sc} \xi=\frac{\mathrm{sn} \xi}{\mathrm{cn} \xi}$

Step 1. Substituting (6) along with (7) into (5) and then setting all coefficients of $x^{p} F^{j}(\xi) F^{\prime q}(\xi)(p, q=0,1 ; j=0,1,2, \ldots)$ of the resulting equation to zero, we get an overdetermined PDEs system for $a_{i}(i=0,1,2, \ldots, k)$ and $\xi$.

Step 2. Solving the set of overdetermined PDEs by use of Mathematica can permit obtention of explicit expressions of $a_{i}(i=0,1,2, \ldots, l)$ and $\xi$.

Step 3. Substituting $a_{i}(i=0,1,2, \ldots, l)$ and $\xi$ obtained in Step 2 into (6) along with the solutions of (7), we can get the exact solutions of (5).

Remark 1. In this paper, we consider the case of $m=4$. As we know the more solutions of (7) we find, the more exact solutions of (5) may be obtained. However, the general solutions are difficult to be listed because of the complexity of (7). Some special solutions [19, 26-29] are listed as follows.

Case 1. Suppose that $h_{1}=h_{3}=0$; the solutions of (7) are given in Tables 1 and 2 .

Case 2. Suppose that $h_{0}=h_{1}=0$; the solutions of (7) are given in Table 3. 
TABLE 2: Solutions of Case 2.1 (continued). $m(0<m<1)$ denotes the modulus of the Jacobi elliptic function, $k_{1}=\sqrt{1-m^{2}}$, and $A, B, C$ $(A B C \neq 0)$, and $D$ are arbitrary constants.

\begin{tabular}{|c|c|c|c|}
\hline$h_{0}$ & $h_{2}$ & $h_{4}$ & $F(\xi)$ \\
\hline 1 & $2 m^{2}-1$ & $-m^{2}\left(1-m^{2}\right)$ & $\operatorname{sd} \xi=\frac{\operatorname{sn} \xi}{\operatorname{dn} \xi}$ \\
\hline $1-m^{2}$ & $2-m^{2}$ & 1 & $\operatorname{cs} \xi=\frac{\mathrm{cn} \xi}{\operatorname{sn} \xi}$ \\
\hline$-m^{2}\left(1-m^{2}\right)$ & $2 m^{2}-1$ & 1 & $\mathrm{ds} \xi=\frac{\mathrm{dn} \xi}{\operatorname{sn} \xi}$ \\
\hline$\underline{1}$ & $1-2 m^{2}$ & $\underline{1}$ & \multirow{2}{*}{$\mathrm{ns} \xi \pm \mathrm{cs} \xi$} \\
\hline$\overline{4}$ & 2 & $\overline{4}$ & \\
\hline $1-m^{2}$ & $1+m^{2}$ & $1-m^{2}$ & \multirow{2}{*}{$\mathrm{nc} \xi \pm \mathrm{sc} \xi$} \\
\hline 4 & 2 & 4 & \\
\hline$m^{2}$ & $m^{2}-2$ & 1 & \multirow{2}{*}{$\mathrm{ns} \xi \pm \mathrm{ds} \xi$} \\
\hline$\overline{4}$ & 2 & $\overline{4}$ & \\
\hline$\underline{m^{2}}$ & $m^{2}-2$ & $m^{2}$ & \multirow{2}{*}{$\operatorname{sn} \xi \pm i \operatorname{cn} \xi, \frac{\operatorname{dn}(\xi)}{\sqrt{1-m^{2}} \operatorname{sn}(\xi) \pm \operatorname{cn}(\xi)}$} \\
\hline 4 & 2 & 4 & \\
\hline 1 & $1-2 m^{2}$ & 1 & \multirow{2}{*}{$m \mathrm{sn} \xi \pm i \operatorname{dn} \xi, \frac{\operatorname{sn}(\xi)}{1 \pm \mathrm{cn}(\xi)}$} \\
\hline$\overline{4}$ & 2 & $\overline{4}$ & \\
\hline 1 & $m^{2}-2$ & $m^{2}$ & \multirow{2}{*}{$\frac{\operatorname{sn}(\xi)}{1 \pm \operatorname{dn}(\xi)}$} \\
\hline$\overline{4}$ & 2 & $\overline{4}$ & \\
\hline$m^{2}-1$ & $m^{2}+1$ & $m^{2}-1$ & \multirow{2}{*}{$\frac{\operatorname{dn}(\xi)}{1 \pm m \operatorname{sn}(\xi)}, m \operatorname{sd}(\xi) \pm \operatorname{nd}(\xi)$} \\
\hline 4 & 2 & 4 & \\
\hline $1-m^{2}$ & $m^{2}+1$ & $1-m^{2}$ & \multirow{2}{*}{$\frac{\mathrm{cn}(\xi)}{1 \pm \operatorname{sn}(\xi)}, \mathrm{nc}(\xi) \pm \mathrm{sc}(\xi)$} \\
\hline 4 & 2 & 4 & \\
\hline 1 & $m^{2}+1$ & $\left(1-m^{2}\right)^{2}$ & $\operatorname{sn}(\xi)$ \\
\hline$\overline{4}$ & 2 & 4 & $\overline{\operatorname{dn}(\xi) \pm \operatorname{cn}(\xi)}$ \\
\hline 1 & $m^{2}-2$ & $m^{4}$ & $\operatorname{cn}(\xi)$ \\
\hline$\overline{4}$ & 2 & $\overline{4}$ & 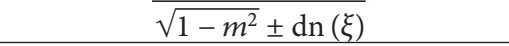 \\
\hline$-\left(1-m^{2}\right)^{2}$ & $m^{2}+1$ & -1 & \multirow{2}{*}{$m \mathrm{cn}(\xi) \pm \operatorname{dn}(\xi)$} \\
\hline 4 & 2 & $\overline{4}$ & \\
\hline 1 & & $\frac{1}{2}$ & \multirow{2}{*}{$\frac{\operatorname{dn}(\xi)}{m \mathrm{mn}(\xi) \pm i \sqrt{1-m^{2}}}$} \\
\hline \multirow[t]{3}{*}{$\overline{4}$} & & $\overline{4}$ & \\
\hline & $1-2 m^{2}$ & & $\operatorname{sn}(\xi)$ \\
\hline & 2 & & $1 \pm \mathrm{cn}(\xi), \sqrt{1-m^{2}} \operatorname{sn}(\xi) \pm \operatorname{dn}(\xi)$ \\
\hline 1 & $2-4 m^{2}$ & 1 & $\frac{\operatorname{sn}(\xi) \mathrm{dn}(\xi)}{\operatorname{cn}(\xi)}$ \\
\hline$(m-1)^{2}$ & $1+m^{2}+6 m$ & $A^{2}(m-1)^{2}$ & $\operatorname{dn}(\xi) \operatorname{cn}(\xi)$ \\
\hline $4 A^{2}$ & 2 & 4 & $\overline{A(1+\operatorname{sn}(\xi))(1+m \operatorname{sn}(\xi))}$ \\
\hline$(m+1)^{2}$ & $1+m^{2}-6 m$ & $A^{2}(m+1)^{2}$ & $\operatorname{dn}(\xi) \operatorname{cn}(\xi)$ \\
\hline $4 A^{2}$ & 2 & 4 & $\overline{A(1+\operatorname{sn}(\xi))(1-m \operatorname{sn}(\xi))}$ \\
\hline \multirow{2}{*}{$-2 m^{3}+m^{4}+m^{2}$} & \multirow{2}{*}{$6 m-m^{2}-1$} & $\underline{-4}$ & $m \mathrm{dn}(\xi) \mathrm{cn}(\xi)$ \\
\hline & & $\bar{m}$ & $1+m \operatorname{sn}^{2}(\xi)$ \\
\hline \multirow{2}{*}{$2 m^{3}+m^{4}+m^{2}$} & \multirow{2}{*}{$-6 m-m^{2}-1$} & $\underline{4}$ & $m \operatorname{dn}(\xi) \mathrm{cn}(\xi)$ \\
\hline & & $\bar{m}$ & $\overline{-1+m \mathrm{sn}^{2}(\xi)}$ \\
\hline \multirow{2}{*}{$2+2 k_{1}-m^{2}$} & & & $m^{2} \operatorname{sn}(\xi) \mathrm{cn}(\xi)$ \\
\hline & $6 \kappa_{1}-m^{2}+2$ & $4 \kappa_{1}$ & $\overline{k_{1}-\operatorname{dn}^{2}(\xi)}$ \\
\hline $2-2 k_{1}-m^{2}$ & $-6 k_{1}-m^{2}+2$ & $-4 k_{1}$ & $-m^{2} \operatorname{sn}(\xi) \mathrm{cn}(\xi)$ \\
\hline $2 \quad 2 n_{1} \quad m$ & $\begin{array}{lll}0 n_{1} & m & 1\end{array}$ & $T n_{1}$ & $k_{1}+\mathrm{dn}^{2}(\xi)$ \\
\hline$m^{2}-1$ & $\underline{m^{2}+1}$ & $\underline{\left(C^{2} m^{2}-B^{2}\right)\left(m^{2}-1\right)}$ & $\sqrt{\left(B^{2}-C^{2}\right) /\left(B^{2}-C^{2} m^{2}\right)+\operatorname{sn}(\xi)}$ \\
\hline $4\left(C^{2} m^{2}-B^{2}\right)$ & 2 & 4 & $B \operatorname{cn}(\xi)+C \operatorname{dn}(\xi)$ \\
\hline$m^{4}$ & $m^{2}$ & $\left(C^{2}+B^{2}\right)$ & $\sqrt{\left(B^{2}+C^{2}-C^{2} m^{2}\right) /\left(B^{2}+C^{2}\right)}+\operatorname{dn}(\xi)$ \\
\hline$\overline{4\left(C^{2}+B^{2}\right)}$ & $\overline{2}$ & 4 & $B \operatorname{sn}(\xi)+C \operatorname{cn}(\xi)$ \\
\hline $2 m-m^{2}-1$ & $2 m^{2}+2$ & $m^{2}-B^{2}-2 B^{2} m$ & $m \mathrm{sn}^{2}(\xi)-1$ \\
\hline$B^{2}$ & $2 m+2$ & $m-D-2 D m$ & $\overline{B\left(m \mathrm{sn}^{2}(\xi)+1\right)}$ \\
\hline
\end{tabular}


TABLE 2: Continued.

\begin{tabular}{lcrr}
\hline$h_{0}$ & $h_{2}$ & $h_{4}$ & $F(\xi)$ \\
\hline$-\frac{2 m+m^{2}+1}{B^{2}}$ & $2 m^{2}+2$ & $-B^{2} m^{2}-B^{2}-2 B^{2} m$ & $\frac{m \mathrm{sn}^{2}(\xi)+1}{B\left(m \mathrm{sn}^{2}(\xi)-1\right)}$ \\
\hline 0 & 1 & $\frac{1}{2}$ & $\pm \frac{\sqrt{2-2 \tanh ^{2}(D-\xi)}}{\tanh (D-\xi)}$ \\
\hline
\end{tabular}

Case 3. If $h_{0}=k, h_{1}=-4 k, h_{2}=-1-k^{2}+6 k, h_{3}=2(k-1)^{2}$, $h_{4}=-(k-1)^{2}$, (7) has the following solutions:

$$
\begin{aligned}
& F(\xi)=\frac{\sqrt{k} \operatorname{sn}(\xi)+1}{2 \sqrt{k} \operatorname{sn}(\xi)+k \operatorname{sn}(\xi)^{2}+1} \\
& F(\xi)=\frac{k^{3 / 2} \operatorname{sn}(\xi)+1}{k^{3 / 2} \operatorname{sn}(\xi)-\operatorname{dn}^{2}(\xi)+1}
\end{aligned}
$$

Case 4. If $h_{0}=-h_{1} / 4, h_{1}<0, h_{2}=-h_{1}\left(2-k^{2}\right), h_{3}=2 h_{1}(1-$ $\left.k^{2}\right), h_{4}=-h_{1}\left(1-k^{2}\right),(7)$ has the following solutions:

$$
\begin{aligned}
& F(\xi)=\frac{\operatorname{sn}\left(\sqrt{-h_{1}} \xi\right)}{\operatorname{sn}\left(\sqrt{-h_{1}} \xi\right)+\operatorname{cn}\left(\sqrt{-h_{1}} \xi\right)+1}, \\
& F(\xi)=\frac{1+\operatorname{cn}\left(\sqrt{-h_{1}} \xi\right)}{-\operatorname{sn}\left(\sqrt{-h_{1}} \xi\right)+\operatorname{cn}\left(\sqrt{-h_{1}} \xi\right)+1} .
\end{aligned}
$$

Case 5. If $h_{0}>0, h_{1}=-4 h_{0}, h_{2}=4 h_{0}\left(2-k^{2}\right), h_{3}=$ $-8 h_{0}\left(1-k^{2}\right), h_{4}=4 h_{0}\left(1-k^{2}\right),(7)$ has the following solutions:

$F(\xi)$

$$
=\frac{\operatorname{cn}\left(2 \sqrt{h_{0}} \xi\right)}{\sqrt{1-k^{2}} \operatorname{sn}\left(2 \sqrt{h_{0}} \xi\right)+\operatorname{cn}\left(2 \sqrt{h_{0}} \xi\right)+\operatorname{dn}\left(2 \sqrt{h_{0}} \xi\right)},
$$

$F(\xi)$

$$
=\frac{\sqrt{1-k^{2}} \operatorname{sn}\left(2 \sqrt{h_{0}} \xi\right)+\operatorname{dn}\left(2 \sqrt{h_{0}} \xi\right)}{\sqrt{1-k^{2}} \operatorname{sn}\left(2 \sqrt{h_{0}} \xi\right)+\operatorname{cn}\left(2 \sqrt{h_{0}} \xi\right)+\operatorname{dn}\left(2 \sqrt{h_{0}} \xi\right)} .
$$

Case 6. If $h_{0}=-h_{1} / 4, h_{1}=-(k \alpha)^{2}, h_{2}=-\left(2 h_{1}+\alpha^{2}\right), h_{3}=$ $2\left(h_{1}+\alpha^{2}\right), h_{4}=-\left(h_{1}+\alpha^{2}\right),(7)$ has the following solutions:

$$
\begin{aligned}
& F(\xi)=\frac{k \operatorname{sn}(\alpha \xi)}{k \operatorname{sn}(\alpha \xi)+\operatorname{dn}(\alpha \xi)-1}, \\
& F(\xi)=\frac{\operatorname{dn}(\alpha \xi)+1}{k \operatorname{sn}(\alpha \xi)-\operatorname{dn}(\alpha \xi)-1}, \\
& F(\xi)=\frac{k \operatorname{cn}(\alpha \xi)}{k \operatorname{cn}(\alpha \xi)+\operatorname{dn}(\alpha \xi)+\sqrt{1-k^{2}}}, \\
& F(\xi)=\frac{\operatorname{dn}(\alpha \xi)+\sqrt{1-k^{2}}}{k \operatorname{cn}(\alpha \xi)+\operatorname{dn}(\alpha \xi)+\sqrt{1-k^{2}}} .
\end{aligned}
$$

\section{Solutions for the Generalized Variable-Coefficient KdV-MKdV Equation}

To solve (1), we first make the transformation

$$
u=u(\xi), \quad \xi=p(t) x+q(t),
$$

where $p(t)$ and $q(t)$ are functions to be determined later. We consider that the solutions of (1) can be expressed as follows:

$$
u(\xi)=\sum_{i=0}^{l} a_{i}(t, x) F^{i}(\xi)
$$

where $a_{i}(t, x)(i=0,1, \ldots, l)$ are functions to be determined later.

Substituting (13) into (1), we can easily find that $l=1$ by balancing $u_{x x x}$ and $u^{2} u_{x}$ in (1). Therefore,

$$
u(\xi)=a_{0}(t, x)+a_{1}(t, x) F(\xi)
$$

With the aid of Mathematica, substituting (14) along with (7) into (1) and then setting each coefficient of $F(\xi)$ to zero, we obtain a set of nonlinear and parameterized partial differential equations with respect to unknowns $a_{0}, a_{1}, p$, and $q$ as follows:

$$
\begin{aligned}
& a_{0 t}+A f_{4}(t) a_{0}=0, \\
& a_{1 t}+A f_{4}(t) a_{1}=0, \\
& -6 f_{0}(t) a_{1}^{2} p(t)-12 p(t) a_{0} a_{1}^{2} \\
& \quad+3 a_{1} h_{3} f_{2}(t) p(t)^{3}=0, \\
& a_{1} q^{\prime}(t)-6 a_{0} a_{1} f_{0}(t) p(t)-6 a_{0}^{2} a_{1} f_{1}(t) p(t) \\
& \quad+a_{1} h_{2} f_{2}(t) p(t)^{3}-a_{1} f_{3}(t) p(t)=0, \\
& a_{1} p^{\prime}(t)+a_{1} f_{4}(t) p(t)=0, \\
& -6 f_{1}(t) a_{1}^{3} p(t)+6 h_{4} f_{2}(t) a_{1} p(t)^{3}=0, \\
& a_{0 x} f_{4}(t)=0, \\
& a_{1 x} f_{4}(t)=0,
\end{aligned}
$$

where $a_{0 t}=\partial a_{0} / \partial t, a_{1 t}=\partial a_{1} / \partial t, a_{0 x}=\partial a_{0} / \partial x, a_{1 x}=\partial a_{1} / \partial x$. 
TABLE 3: Solutions of Case 2.2. $\Delta_{2}=h_{3}^{2}-4 h_{2} h_{4}, \epsilon= \pm 1$, and $a, b, c$, and $d$ are the arbitrary constants.

\begin{tabular}{|c|c|c|c|c|}
\hline$h_{2}$ & $h_{3}$ & $h_{4}$ & $\Delta_{2}$ & $F(\xi)$ \\
\hline \multirow{2}{*}{1} & $2 c$ & $c^{2}-b^{2}$ & & $a \operatorname{sech}(\xi)$ \\
\hline & $\bar{a}$ & $\overline{a^{2}}$ & & $\overline{b+c \operatorname{sech}(\xi)}$ \\
\hline \multirow{2}{*}{1} & $2 c$ & $c^{2}+b^{2}$ & & $a \operatorname{csch}(\xi)$ \\
\hline & $\bar{a}$ & $a^{2}$ & & $\overline{b+c \operatorname{csch}(\xi)}$ \\
\hline \multirow{2}{*}{4} & $-\frac{4(2 b+d)}{-}$ & $c^{2}+4 b^{2}+4 b d$ & & $a \operatorname{sech}^{2}(\xi)$ \\
\hline & $a$ & $a^{2}$ & & $\overline{b \operatorname{sech}^{2}(\xi)+c \tanh (\xi)+d}$ \\
\hline \multirow{2}{*}{4} & $4(d-2 b)$ & $c^{2}+4 b^{2}-4 b d$ & & $\operatorname{acsch}^{2}(\xi)$ \\
\hline & $a$ & $a^{2}$ & & $\overline{b \operatorname{csch}^{2}(\xi)+c \operatorname{coth}(\xi)+d}$ \\
\hline \multirow{2}{*}{$a^{2}$} & $2 a b$ & $b^{2}$ & & $-a c$ \\
\hline & 200 & 0 & & $\overline{b(c+\cosh (a \xi)-\sinh (a \xi))}$ \\
\hline \multirow{2}{*}{$a^{2}$} & $2 a h$ & $h^{2}$ & & $a(\cosh (a \xi)+\sinh (a \xi))$ \\
\hline & $2 a 0$ & 0 & & $b(c+\cosh (a \xi)+\sinh (a \xi))$ \\
\hline \multirow{2}{*}{-1} & $\underline{2 c}$ & $c^{2}-b^{2}$ & & $\operatorname{asec}(\xi)$ \\
\hline & $\bar{a}$ & $a^{2}$ & & $b+c \sec (\xi)$ \\
\hline \multirow{2}{*}{-1} & $\underline{2 c}$ & $-c^{2}-b^{2}$ & & $\operatorname{acsc}(\xi)$ \\
\hline & $\bar{a}$ & $\overline{a^{2}}$ & & $\overline{b+c \csc (\xi)}$ \\
\hline \multirow{2}{*}{-4} & $4(2 b+d)$ & $-c^{2}+4 b^{2}+4 b d$ & & $\operatorname{asec}^{2}(\xi)$ \\
\hline & $a$ & $a^{2}$ & & $\overline{b \sec ^{2}(\xi)+c \tan (\xi)+d}$ \\
\hline \multirow{2}{*}{-4} & $4(2 b+d)$ & $-c^{2}+4 b^{2}+4 b d$ & & $a \csc ^{2}(\xi)$ \\
\hline & $a$ & $a^{2}$ & & $\overline{b \csc ^{2}(\xi)+c \cot (\xi)+d}$ \\
\hline \multirow{2}{*}{$>0$} & & & & $-h_{2} h_{3} \operatorname{sech}^{2}\left(\left(\sqrt{h_{2}} / 2\right) \xi\right)$ \\
\hline & & & & $\overline{h_{3}^{2}-h_{2} h_{4}\left(1+\epsilon \tanh \left(\left(\sqrt{h_{2}} / 2\right) \xi\right)\right)^{2}}$ \\
\hline \multirow{2}{*}{$>0$} & & & & $h_{2} h_{3} \operatorname{csch}^{2}\left(\left(\sqrt{h_{2}} / 2\right) \xi\right)$ \\
\hline & & & & $\overline{h_{3}^{2}-h_{2} h_{4}\left(1+\epsilon \operatorname{coth}\left(\left(\sqrt{h_{2}} / 2\right) \xi\right)\right)^{2}}$ \\
\hline \multirow{2}{*}{$>0$} & & & & $4 h_{2} e^{\epsilon \sqrt{h_{2}} \xi}$ \\
\hline & & & & $\overline{\left(e^{\left.\epsilon \sqrt{h_{2} \xi}\right)^{2}-4 h_{2} h_{4}}\right.}$ \\
\hline \multirow{2}{*}{$>0$} & & & $>0$ & $2 h_{2} \operatorname{sech}\left(\sqrt{h_{2}} \xi\right)$ \\
\hline & & & $>0$ & $\overline{\epsilon \sqrt{\Delta_{2}}-h_{3} \operatorname{sech}\left(\sqrt{h_{2}} \xi\right)}$ \\
\hline \multirow{2}{*}{$<0$} & & & & $2 h_{2} \sec \left(\sqrt{-h_{2}} \xi\right)$ \\
\hline & & & $>0$ & $\overline{\epsilon \sqrt{\Delta_{2}}-h_{3} \sec \left(\sqrt{-h_{2}} \xi\right)}$ \\
\hline \multirow{2}{*}{$>0$} & & & $<0$ & $2 h_{2} \operatorname{csch}\left(\sqrt{h_{2}} \xi\right)$ \\
\hline & & & $<0$ & $\epsilon \sqrt{-\Delta_{2}}-h_{3} \operatorname{csch}\left(\sqrt{h_{2}} \xi\right)$ \\
\hline \multirow{2}{*}{$<0$} & & & $>0$ & $2 h_{2} \csc \left(\sqrt{-h_{2}} \xi\right)$ \\
\hline & & & $>0$ & $\epsilon \sqrt{\Delta_{2}}-h_{3} \csc \left(\sqrt{-h_{2}} \xi\right)$ \\
\hline$>0$ & & & $=0$ & $-\frac{h_{2}}{h_{3}}\left[1 \pm \tanh \left(\frac{\sqrt{h_{2}} \xi}{2}\right)\right]$ \\
\hline$>0$ & & & $=0$ & $-\frac{h_{2}}{h_{3}}\left[1 \pm \operatorname{coth}\left(\frac{\sqrt{h_{2}} \xi}{2}\right)\right]$ \\
\hline \multirow{2}{*}{$>0$} & & $>0$ & & $-h_{2} \operatorname{sech}^{2}\left(\left(\sqrt{h_{2}} / 2\right) \xi\right)$ \\
\hline & & $>0$ & & $\overline{h_{3}+2 \epsilon \sqrt{h_{2} h_{4}} \tanh \left(\left(\sqrt{h_{2}} / 2\right) \xi\right)}$ \\
\hline \multirow{2}{*}{$>0$} & & $>0$ & & $h_{2} \operatorname{csch}^{2}\left(\left(\sqrt{h_{2}} / 2\right) \xi\right)$ \\
\hline & & $>0$ & & $\overline{h_{3}+2 \epsilon \sqrt{h_{2} h_{4}} \operatorname{coth}\left(\left(\sqrt{h_{2}} / 2\right) \xi\right)}$ \\
\hline \multirow{2}{*}{$<0$} & & $>0$ & & $-h_{2} \sec ^{2}\left(\left(\sqrt{-h_{2}} / 2\right) \xi\right)$ \\
\hline & & $>0$ & & $\overline{h_{3}+2 \epsilon \sqrt{-h_{2} h_{4}} \tanh \left(\left(\sqrt{-h_{2}} / 2\right) \xi\right)}$ \\
\hline \multirow{2}{*}{$<0$} & & & & $-h_{2} \csc ^{2}\left(\left(\sqrt{-h_{2}} / 2\right) \xi\right)$ \\
\hline & & $>0$ & & $\overline{h_{3}+2 \epsilon \sqrt{-h_{2} h_{4}} \cot \left(\left(\sqrt{-h_{2}} / 2\right) \xi\right)}$ \\
\hline$>0$ & & $=0$ & & $\pm 4 h_{2} e^{\epsilon \sqrt{h_{2}} \xi}$ \\
\hline 20 & & -0 & & $1-4 h_{2} h_{4} e^{2 \epsilon \sqrt{h_{2} \xi}}$ \\
\hline
\end{tabular}


For the sake of simplicity, in the rest of this paper, we introduce four notations:

$$
\begin{aligned}
& \varphi(t)=\exp \left[-\int^{t} f_{4}(s) d s\right], \\
& \varphi(s)=\exp \left[-\int^{s} f_{4}(\tau) d \tau\right], \\
& \phi(t)=\exp \left[-\int^{t} A f_{4}(s) d s\right], \\
& \phi(s)=\exp \left[-\int^{s} A f_{4}(\tau) d \tau\right] .
\end{aligned}
$$

Solving the system by Mathematica, we get the following solution set:

$$
\begin{aligned}
& a_{0}=-\frac{a_{1}\left[2 a_{1} f_{0}(t)-p^{2}(t) h_{3} f_{2}(t)\right]}{4 p^{2}(t) h_{4} f_{2}(t)} \\
& a_{1}=c_{2} \phi(t) \\
& p(t)=c_{1} \varphi(t) \\
& f_{1}(t)=\frac{h_{4} p^{2}(t) f_{2}(t)}{a_{1}^{2}} \\
& q(t)=c_{3}+\int \frac{1}{8 h_{4} f_{2}(s) p(s)}\left[-12 a_{1}^{2} f_{0}^{2}(s)\right. \\
& +\left(3 h_{3}^{2}-8 h_{2} h_{4}\right) p^{4}(s) f_{2}^{2}(s) \\
& \left.+8 h_{4} p^{2}(s) f_{2}(s) f_{3}(s)\right] d s
\end{aligned}
$$

where $c_{1}, c_{2}$, and $c_{3}$ are arbitrary constants.

Therefore, from Cases 1-6, we obtain many kinds of explicit nontraveling solutions of (1). Taking Case 1 as an example, we have the following.

Case 7. Soliton and soliton-like solutions are as follows (Figures 1 and 2):

$$
u_{1}=-\frac{a_{1}^{2} f_{0}(t)}{2 p^{2}(t) h_{4} f_{2}(t)}+c_{2} \phi(t) \sqrt{\frac{-h_{2}}{h_{4}}} \operatorname{sech}\left(\sqrt{h_{2}} \xi\right),
$$

where $\xi=c_{1} \varphi(t) x+\int\left(\left[-3 a_{1}^{2} f_{0}^{2}(s)-2 h_{2} h_{4} p^{4}(s) f_{2}^{2}(s)+\right.\right.$ $\left.\left.2 h_{4} p^{2}(s) f_{2}(s) f_{3}(s)\right] / 2 h_{4} f_{2}(s) p(s)\right) d s+c_{3}$. Consider

$$
u_{2}=-\frac{a_{1}^{2} f_{0}(t)}{2 p^{2}(t) h_{4} f_{2}(t)}+c_{2} \phi(t) \sqrt{\frac{h_{2}}{h_{4}}} \operatorname{csch}\left(\sqrt{h_{2}} \xi\right),
$$

where $\xi=c_{1} \varphi(t) x+\int\left(\left[-3 a_{1}^{2} f_{0}^{2}(s)-2 h_{2} h_{4} p^{4}(s) f_{2}^{2}(s)+\right.\right.$ $\left.\left.2 h_{4} p^{2}(s) f_{2}(s) f_{3}(s)\right] / 2 h_{4} f_{2}(s) p(s)\right) d s+c_{3}$. Consider

$$
\begin{aligned}
u_{3}= & -\frac{a_{1}^{2} f_{0}(t)}{2 p^{2}(t) h_{4} f_{2}(t)} \\
& +c_{2} \phi(t) \sqrt{\frac{-h_{2}}{2 h_{4}}} \tanh \left(\sqrt{\frac{-h_{2}}{2} \xi}\right),
\end{aligned}
$$

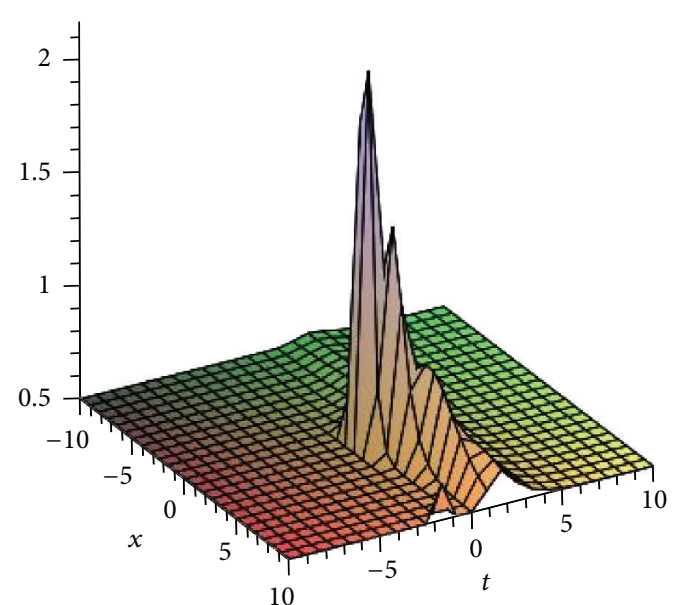

FIGURE 1: The soliton-like solution (18) with $h_{2}=c_{1}=c_{2}=A=1$, $h_{4}=-1, c_{3}=0$, and $f_{0}(t)=f_{2}(t)=f_{3}(t)=t ; f_{1}(t)=-t$.

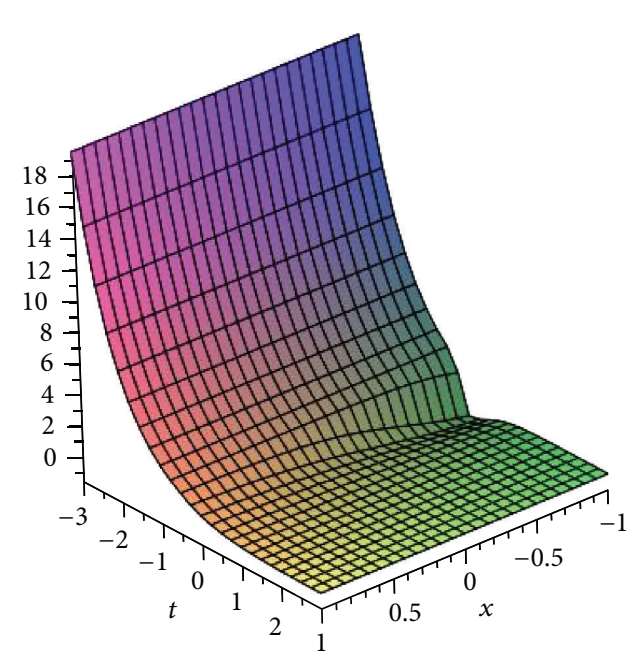

FIgURE 2: The soliton-like solution (20) with $h_{2}=-2, h_{4}=h_{0}=$ $c_{1}=c_{2}=A=1, c_{3}=0$, and $f_{0}(t)=f_{1}(t)=f_{2}(t)=f_{3}(t)=t$.

where $\xi=c_{1} \varphi(t) x+\int\left(\left[-3 a_{1}^{2} f_{0}^{2}(s)-2 h_{2} h_{4} p^{4}(s) f_{2}^{2}(s)+\right.\right.$ $\left.\left.2 h_{4} p^{2}(s) f_{2}(s) f_{3}(s)\right] / 2 h_{4} f_{2}(s) p(s)\right) d s+c_{3}$. Consider

$$
u_{4}=-\frac{a_{1}^{2} f_{0}(t)}{p^{2}(t) f_{2}(t)} \pm c_{2} \phi(t) \frac{\sqrt{2-2 \tanh ^{2}(D-\xi)}}{\tanh (D-\xi)}
$$

where $\xi=c_{1} \varphi(t) x+\int\left(1 / 2 h_{4} f_{2}(s) p(s)\right)\left[-3 a_{1}^{2} f_{0}^{2}(s)-\right.$ $\left.p^{4}(s) f_{2}^{2}(s)+p^{2}(s) f_{2}(s) f_{3}(s)\right] d s+c_{3}$.

Case 8. Triangular periodic solutions are as follows:

$$
u_{5}=-\frac{a_{1}^{2} f_{0}(t)}{2 p^{2}(t) h_{4} f_{2}(t)}+c_{2} \phi(t) \sqrt{\frac{-h_{2}}{h_{4}}} \sec \left(\sqrt{-h_{2}} \xi\right) \text {, }
$$


where $\xi=c_{1} \varphi(t) x+\int\left(1 / 2 h_{4} f_{2}(s) p(s)\right)\left[-3 a_{1}^{2} f_{0}^{2}(s)-\right.$ $\left.2 h_{2} h_{4} p^{4}(s) f_{2}^{2}(s)+2 h_{4} p^{2}(s) f_{2}(s) f_{3}(s)\right] d s+c_{3}$. Consider

$$
u_{6}=-\frac{a_{1}^{2} f_{0}(t)}{2 p^{2}(t) h_{4} f_{2}(t)}+c_{2} \phi(t) \sqrt{\frac{h_{2}}{h_{4}}} \csc \left(\sqrt{-h_{2}} \xi\right),
$$

where $\xi=c_{1} \varphi(t) x+\int\left(1 / 2 h_{4} f_{2}(s) p(s)\right)\left[-3 a_{1}^{2} f_{0}^{2}(s)-\right.$ $\left.2 h_{2} h_{4} p^{4}(s) f_{2}^{2}(s)+2 h_{4} p^{2}(s) f_{2}(s) f_{3}(s)\right] d s+c_{3}$. Consider

$$
\begin{aligned}
u_{7}= & -\frac{a_{1}^{2} f_{0}(t)}{2 p^{2}(t) h_{4} f_{2}(t)} \\
& +c_{2} \phi(t) \sqrt{\frac{-h_{2}}{2 h_{4}}} \tan \left(\sqrt{\frac{-h_{2}}{2}} \xi\right),
\end{aligned}
$$

where $\xi=c_{1} \varphi(t) x+\int\left(1 / 2 h_{4} f_{2}(s) p(s)\right)\left[-3 a_{1}^{2} f_{0}^{2}(s)-\right.$ $\left.2 h_{2} h_{4} p^{4}(s) f_{2}^{2}(s)+2 h_{4} p^{2}(s) f_{2}(s) f_{3}(s)\right] d s+c_{3}$.

Case 9. Jacobi elliptic function solutions and combined Jacobi elliptic function solutions are as follows:

$$
u_{8}=-\frac{a_{1}^{2} f_{0}(t)}{2 p^{2}(t) m^{2} f_{2}(t)}+c_{2} \phi(t) \operatorname{cd}(\xi)
$$

where $\xi=c_{1} \varphi(t) x+\int\left(\left[-3 a_{1}^{2} f_{0}^{2}(s)+2\left(m^{4}+m^{2}\right) p^{4}(s) f_{2}^{2}(s)+\right.\right.$ $\left.\left.2 m^{2} p^{2}(s) f_{2}(s) f_{3}(s)\right] / 2 m^{2} f_{2}(s) p(s)\right) d s+c_{3}$. Consider

$$
u_{9}=-\frac{a_{1}^{2} f_{0}(t)}{2 p^{2}(t) f_{2}(t)}+c_{2} \phi(t) \mathrm{ns}(\xi)
$$

where $\xi=c_{1} \varphi(t) x+\int\left(\left[-3 a_{1}^{2} f_{0}^{2}(s)+2\left(m^{2}+1\right) p^{4}(s) f_{2}^{2}(s)+\right.\right.$ $\left.\left.2 p^{2}(s) f_{2}(s) f_{3}(s)\right] / 2 f_{2}(s) p(s)\right) d s+c_{3}$. Consider

$$
u_{10}=\frac{a_{1}^{2} f_{0}(t)}{2 p^{2}(t) f_{2}(t)}+c_{2} \phi(t) \operatorname{dn}(\xi)
$$

where $\xi=c_{1} \varphi(t) x+\int\left(\left[3 a_{1}^{2} f_{0}^{2}(s)+2\left(m^{2}-2\right) p^{4}(s) f_{2}^{2}(s)+\right.\right.$ $\left.\left.2 p^{2}(s) f_{2}(s) f_{3}(s)\right] / 2 f_{2}(s) p(s)\right) d s+c_{3}$. Consider

$$
u_{11}=\frac{a_{1}^{2} f_{0}(t)}{2 m^{2} p^{2}(t) f_{2}(t)}+c_{2} \phi(t) \operatorname{cn}(\xi),
$$

where $\xi=c_{1} \varphi(t) x+\int\left(\left[3 a_{1}^{2} f_{0}^{2}(s)+2\left(m^{2}-2 m^{4}\right) p^{4}(s) f_{2}^{2}(s)+\right.\right.$ $\left.\left.2 m^{2} p^{2}(s) f_{2}(s) f_{3}(s)\right] / 2 m^{2} f_{2}(s) p(s)\right) d s+c_{3}$. Consider

$$
u_{12}=-\frac{a_{1}^{2} f_{0}(t)}{2 p^{2}(t)\left(1-m^{2}\right) f_{2}(t)}+c_{2} \phi(t) \mathrm{nc}(\xi)
$$

where $\xi=c_{1} \varphi(t) x+\int\left(\left[-3 a_{1}^{2} f_{0}^{2}(s)+\left(4 m^{4}-6 m^{2}+2\right) p^{4}(s) f_{2}^{2}(s)+\right.\right.$ $\left.\left.\left(2-2 m^{2}\right) p^{2}(s) f_{2}(s) f_{3}(s)\right] / 2\left(1-m^{2}\right) f_{2}(s) p(s)\right) d s+c_{3}$. Consider

$$
u_{13}=-\frac{a_{1}^{2} f_{0}(t)}{2 p^{2}(t)\left(m^{2}-1\right) f_{2}(t)}+c_{2} \phi(t) \operatorname{nd}(\xi)
$$

where $\xi=c_{1} \varphi(t) x+\int\left(\left[-3 a_{1}^{2} f_{0}^{2}(s)+2\left(m^{4}-3 m^{2}+2\right) p^{4}(s) f_{2}^{2}(s)+\right.\right.$ $\left.\left.\left(2 m^{2}-2\right) p^{2}(s) f_{2}(s) f_{3}(s)\right] /\left(2 m^{2}-2\right) f_{2}(s) p(s)\right) d s+c_{3}$. Consider

$$
u_{14}=-\frac{a_{1}^{2} f_{0}(t)}{2 p^{2}(t) f_{2}(t)}+c_{2} \phi(t) \operatorname{cs}(\xi),
$$

where $\xi=c_{1} \varphi(t) x+\int\left(\left[-3 a_{1}^{2} f_{0}^{2}(s)+\left(2 m^{2}-4\right) p^{4}(s) f_{2}^{2}(s)+\right.\right.$ $\left.\left.2 p^{2}(s) f_{2}(s) f_{3}(s)\right] / 2 f_{2}(s) p(s)\right) d s+c_{3}$. Consider

$$
u_{15}=\frac{a_{1}^{2} f_{0}(t)}{2 p^{2}(t)\left(-1+m^{2}\right) f_{2}(t)}+c_{2} \phi(t) \operatorname{sc}(\xi),
$$

where $\xi=c_{1} \varphi(t) x+\int\left(\left[-3 a_{1}^{2} f_{0}^{2}(s)-2\left(m^{4}-3 m^{2}+2\right) p^{4}(s) f_{2}^{2}(s)+\right.\right.$ $\left.\left.\left(2-2 m^{2}\right) p^{2}(s) f_{2}(s) f_{3}(s)\right] / 2\left(1-m^{2}\right) f_{2}(s) p(s)\right) d s+c_{3}$. Consider

$$
u_{16}=-\frac{a_{1}^{2} f_{0}(t)}{2 p^{2}(t) m^{2}\left(m^{2}-1\right) f_{2}(t)}+c_{2} \phi(t) \operatorname{sd}(\xi),
$$

where $\xi=c_{1} \varphi(t) x+\int\left(\left[-3 a_{1}^{2} f_{0}^{2}(s)-2 m^{2}\left(2 m^{4}-3 m^{2}+\right.\right.\right.$ 1) $\left.p^{4}(s) f_{2}^{2}(s)+2 m^{2}\left(m^{2}-1\right) p^{2}(s) f_{2}(s) f_{3}(s)\right] / 2 m^{2}\left(m^{2}-\right.$ 1) $\left.f_{2}(s) p(s)\right) d s+c_{3}$. Consider

$$
u_{17}=-\frac{a_{1}^{2} f_{0}(t)}{2 p^{2}(t) f_{2}(t)}+c_{2} \phi(t) \mathrm{ds}(\xi),
$$

where $\xi=c_{1} \varphi(t) x+\int\left(\left[-3 a_{1}^{2} f_{0}^{2}(s)-2\left(2 m^{2}-1\right) p^{4}(s) f_{2}^{2}(s)+\right.\right.$ $\left.\left.2 p^{2}(s) f_{2}(s) f_{3}(s)\right] / 2 f_{2}(s) p(s)\right) d s+c_{3}$. Consider

$$
u_{18}=-\frac{2 a_{1}^{2} f_{0}(t)}{p^{2}(t) f_{2}(t)}+c_{2} \phi(t)[\mathrm{ns}(\xi) \pm \mathrm{cs}(\xi)],
$$

where $\xi=c_{1} \varphi(t) x+\int\left(\left[-12 a_{1}^{2} f_{0}^{2}(s)+\left(2 m^{2}-1\right) p^{4}(s) f_{2}^{2}(s)+\right.\right.$ $\left.\left.2 p^{2}(s) f_{2}(s) f_{3}(s)\right] / 2 f_{2}(s) p(s)\right) d s+c_{3}$. Consider

$$
\begin{aligned}
u_{19}= & \frac{2 a_{1}^{2} f_{0}(t)}{p^{2}(t)\left(m^{2}-1\right) f_{2}(t)} \\
& +c_{2} \phi(t)[\operatorname{nc}(\xi) \pm \operatorname{sc}(\xi)],
\end{aligned}
$$

where $\xi=c_{1} \varphi(t) x+\int\left(\left[-12 a_{1}^{2} f_{0}^{2}(s)+\left(m^{4}-1\right) p^{4}(s) f_{2}^{2}(s)+2(1-\right.\right.$ $\left.\left.\left.m^{2}\right) p^{2}(s) f_{2}(s) f_{3}(s)\right] / 2\left(1-m^{2}\right) f_{2}(s) p(s)\right) d s+c_{3}$. Consider

$$
u_{20}=-\frac{2 a_{1}^{2} f_{0}(t)}{p^{2}(t) f_{2}(t)}+c_{2} \phi(t)[\mathrm{ns}(\xi)+\mathrm{ds}(\xi)],
$$

where $\xi=c_{1} \varphi(t) x+\int\left(\left[-12 a_{1}^{2} f_{0}^{2}(s)-\left(m^{2}-1\right) p^{4}(s) f_{2}^{2}(s)+\right.\right.$ $\left.\left.2 p^{2}(s) f_{2}(s) f_{3}(s)\right] / 2 f_{2}(s) p(s)\right) d s+c_{3}$. Consider

$$
u_{21}=-\frac{2 a_{1}^{2} f_{0}(t)}{p^{2}(t) m^{2} f_{2}(t)}+c_{2} \phi(t)[\operatorname{sn}(\xi) \pm i \mathrm{cn}(\xi)],
$$

where $\xi=c_{1} \varphi(t) x+\int\left(\left[-12 a_{1}^{2} f_{0}^{2}(s)-\left(m^{4}-2 m^{2}\right) p^{4}(s) f_{2}^{2}(s)+\right.\right.$ $\left.\left.2 m^{2} p^{2}(s) f_{2}(s) f_{3}(s)\right] / 2 m^{2} f_{2}(s) p(s)\right) d s+c_{3}$. Consider

$$
\begin{aligned}
u_{22}= & -\frac{2 a_{1}^{2} f_{0}(t)}{p^{2}(t) m^{2} f_{2}(t)} \\
& +c_{2} \phi(t) \frac{\operatorname{dn}(\xi)}{i \sqrt{1-m^{2}} \operatorname{sn}(\xi) \pm \mathrm{cn}(\xi)},
\end{aligned}
$$


where $\xi=c_{1} \varphi(t) x+\int\left(\left[-12 a_{1}^{2} f_{0}^{2}(s)-\left(m^{4}-2 m^{2}\right) p^{4}(s) f_{2}^{2}(s)+\right.\right.$ $\left.\left.2 m^{2} p^{2}(s) f_{2}(s) f_{3}(s)\right] / 2 m^{2} f_{2}(s) p(s)\right) d s+c_{3}$. Consider

$$
u_{23}=-\frac{a_{1}^{2} f_{0}(t)}{2 p^{2}(t) f_{2}(t)}+c_{2} \phi(t) \frac{\operatorname{sn}(\xi) \mathrm{dn}(\xi)}{\mathrm{cn}(\xi)},
$$

where $\xi=c_{1} \varphi(t) x+\int\left(\left[-3 a_{1}^{2} f_{0}^{2}(s)-2\left(2-4 m^{2}\right) p^{4}(s) f_{2}^{2}(s)+\right.\right.$ $\left.\left.2 p^{2}(s) f_{2}(s) f_{3}(s)\right] / 2 f_{2}(s) p(s)\right) d s+c_{3}$. Consider

$$
\begin{aligned}
u_{24}= & -\frac{2 a_{1}^{2} f_{0}(t)}{p^{2}(t) A^{2}\left(-1+m^{2}\right) f_{2}(t)} \\
& +c_{2} \phi(t) \frac{\operatorname{dn}(\xi) \operatorname{cn}(\xi)}{A(1+\operatorname{sn}(\xi))(1+m s n(\xi))},
\end{aligned}
$$

where $\xi=c_{1} \varphi(t) x+\int\left(\left[-12 a_{1}^{2} f_{0}^{2}(s)-\left(-1+m^{2}\right)\left(m^{2}+\right.\right.\right.$ $\left.6 m+1) p^{4}(s) f_{2}^{2}(s)+2 A^{2}\left(-1+m^{2}\right) p^{2}(s) f_{2}(s) f_{3}(s)\right] / 2 A^{2}(-1+$ $\left.\left.m^{2}\right) f_{2}(s) p(s)\right) d s+c_{3}$. Consider

$$
\begin{aligned}
u_{25}= & -\frac{2 a_{1}^{2} f_{0}(t)}{p^{2}(t) A^{2}\left(1+m^{2}\right) f_{2}(t)} \\
& +c_{2} \phi(t) \frac{\operatorname{dn}(\xi) \operatorname{cn}(\xi)}{A(1+\operatorname{sn}(\xi))(1-m \operatorname{sn}(\xi))},
\end{aligned}
$$

where $\xi=c_{1} \varphi(t) x+\int\left(\left[-12 a_{1}^{2} f_{0}^{2}(s)-\left(1+m^{2}\right)\left(m^{2}-\right.\right.\right.$ $\left.6 m+1) p^{4}(s) f_{2}^{2}(s)+2 A^{2}\left(1+m^{2}\right) p^{2}(s) f_{2}(s) f_{3}(s)\right] / 2 A^{2}(1+$ $\left.\left.m^{2}\right) f_{2}(s) p(s)\right) d s+c_{3}$. Consider

$$
u_{26}=\frac{m a_{1}^{2} f_{0}(t)}{8 p^{2}(t) f_{2}(t)}+c_{2} \phi(t) \frac{m \mathrm{dn}(\xi) \mathrm{cn}(\xi)}{1+m \mathrm{sn}^{2}(\xi)}
$$

where $\xi=c_{1} \varphi(t) x+\int\left(m^{2}\left[3 m a_{1}^{2} f_{0}^{2}(s)-8\left(m^{2}-6 m+\right.\right.\right.$ 1) $\left.\left.p^{4}(s) f_{2}^{2}(s)+8 p^{2}(s) f_{2}(s) f_{3}(s)\right] / 8 f_{2}(s) p(s)\right) d s+c_{3}$. Consider

$$
u_{27}=-\frac{m a_{1}^{2} f_{0}(t)}{8 p^{2}(t) f_{2}(t)}+c_{2} \phi(t) \frac{m \mathrm{dn}(\xi) \mathrm{cn}(\xi)}{m \mathrm{sn}^{2}(\xi)-1}
$$

where $\xi=c_{1} \varphi(t) x+\int\left(m^{2}\left[-3 m a_{1}^{2} f_{0}^{2}(s)+8\left(m^{2}+6 m+\right.\right.\right.$ 1) $\left.\left.p^{4}(s) f_{2}^{2}(s)-8 p^{2}(s) f_{2}(s) f_{3}(s)\right] / 8 f_{2}(s) p(s)\right) d s+c_{3}$. Consider

$$
u_{28}=-\frac{a_{1}^{2} f_{0}(t)}{8 p^{2}(t) k_{1} f_{2}(t)}+c_{2} \phi(t) \frac{m^{2} \operatorname{sn}(\xi) \mathrm{cn}(\xi)}{k_{1}-\operatorname{dn}^{2}(\xi)},
$$

where $\xi=c_{1} \varphi(t) x+\int\left(\left[-3 a_{1}^{2} f_{0}^{2}(s)-8 k_{1}\left(6 k_{1}-m^{2}+\right.\right.\right.$ 2) $\left.\left.p^{4}(s) f_{2}^{2}(s)+8 k_{1} p^{2}(s) f_{2}(s) f_{3}(s)\right] / 8 k_{1} f_{2}(s) p(s)\right) d s+c_{3}$. Consider

$$
u_{29}=\frac{a_{1}^{2} f_{0}(t)}{8 k_{1} p^{2}(t) f_{2}(t)}-c_{2} \phi(t) \frac{m^{2} \operatorname{sn}(\xi) \operatorname{cn}(\xi)}{k_{1}+\operatorname{dn}^{2}(\xi)},
$$

where $\xi=c_{1} \varphi(t) x+\int\left(\left[3 a_{1}^{2} f_{0}^{2}(s)+8 k_{1}\left(6 k_{1}+m^{2}-2\right) p^{4}(s) f_{2}^{2}(s)+\right.\right.$ $\left.\left.8 k_{1} p^{2}(s) f_{2}(s) f_{3}(s)\right] / 8 k_{1} f_{2}(s) p(s)\right) d s+c_{3}$. Consider

$$
\begin{aligned}
u_{30}= & -\frac{2 a_{1}^{2} f_{0}(t)}{p^{2}(t)\left(m^{2}-1\right)\left(C^{2} m^{2}-B^{2}\right) f_{2}(t)} \\
& +c_{2} \phi(t) \frac{\sqrt{\left(B^{2}-C^{2}\right) /\left(B^{2}-C^{2} m^{2}\right)}+\operatorname{sn} \xi}{B \operatorname{cn}(\xi)+C \operatorname{dn}(\xi)},
\end{aligned}
$$

where $\xi=c_{1} \varphi(t) x+\int\left(\left[-12 a_{1}^{2} f_{0}^{2}(s)-\left(m^{4}-1\right)\left(C^{2} m^{2}-\right.\right.\right.$ $\left.\left.B^{2}\right) p^{4}(s) f_{2}^{2}(s)+2\left(m^{2}-1\right)\left(C^{2} m^{2}-B^{2}\right) p^{2}(s) f_{2}(s) f_{3}(s)\right] /\left(m^{2}-\right.$ 1) $\left.\left(C^{2} m^{2}-B^{2}\right) f_{2}(s) p(s)\right) d s+c_{3}$. Consider

$$
\begin{aligned}
u_{31} & \\
= & -\frac{2 a_{1}^{2} f_{0}(t)}{p^{2}(t)\left(C^{2}+B^{2}\right) f_{2}(t)} \\
& +c_{2} \phi(t) \frac{\sqrt{\left(B^{2}+C^{2}-C^{2} m^{2}\right) /\left(B^{2}+C^{2}\right)}+\operatorname{dn} \xi}{B \operatorname{sn}(\xi)+C \operatorname{cn}(\xi)},
\end{aligned}
$$

where $\xi=c_{1} \varphi(t) x+\int\left(\left[-12 a_{1}^{2} f_{0}^{2}(s)-\left(C^{2}+B^{2}\right)\left(m^{2}-\right.\right.\right.$ 2) $\left.p^{4}(s) f_{2}^{2}(s)+2\left(C^{2}+B^{2}\right) p^{2}(s) f_{2}(s) f_{3}(s)\right] /\left(C^{2}+\right.$ $\left.\left.B^{2}\right) f_{2}(s) p(s)\right) d s+c_{3}$. Consider

$$
\begin{aligned}
u_{32}= & \frac{a_{1}^{2} f_{0}(t)}{2 p^{2}(t) B^{2}(m+1)^{2} f_{2}(t)} \\
& +c_{2} \phi(t) \frac{m \mathrm{sn}^{2}-1}{B\left(m \mathrm{sn}^{2}+1\right)},
\end{aligned}
$$

where $\xi=c_{1} \varphi(t) x+\int\left(\left[3 a_{1}^{2} f_{0}^{2}(s)-4 B^{2}(m+1)^{2}\left(m^{2}+\right.\right.\right.$ 1) $\left.p^{4}(s) f_{2}^{2}(s)+2 B^{2}(m+1)^{2} p^{2}(s) f_{2}(s) f_{3}(s)\right] / 2 B^{2}(m+$ $\left.1)^{2} f_{2}(s) p(s)\right) d s+c_{3}$. Consider

$$
\begin{aligned}
u_{33}= & \frac{a_{1}^{2} f_{0}(t)}{2 p^{2}(t) B^{2}(m-1)^{2} f_{2}(t)} \\
& +c_{2} \phi(t) \frac{m \mathrm{sn}^{2}+1}{B\left(m \mathrm{sn}^{2}-1\right)},
\end{aligned}
$$

where $\xi=c_{1} \varphi(t) x+\int\left(\left[3 a_{1}^{2} f_{0}^{2}(s)-4 B^{2}(m-1)^{2}\left(m^{2}+\right.\right.\right.$ 1) $\left.p^{4}(s) f_{2}^{2}(s)+2 B^{2}(m-1)^{2} p^{2}(s) f_{2}(s) f_{3}(s)\right] / 2 B^{2}(m-$ $\left.1)^{2} f_{2}(s) p(s)\right) d s+c_{3}$. Consider

$$
\begin{aligned}
u_{34}= & -\frac{2 a_{1}^{2} f_{0}(t)}{p^{2}(t) f_{2}(t)} \\
& +c_{2} \phi(t) \operatorname{dn}(\xi)\left[m \operatorname{cn}(\xi) \pm i \sqrt{1-m^{2}}\right],
\end{aligned}
$$

where $\xi=c_{1} \varphi(t) x+\int\left(\left[-12 a_{1}^{2} f_{0}^{2}(s)+\left(2 m^{2}-1\right) p^{4}(s) f_{2}^{2}(s)+\right.\right.$ $\left.\left.2 p^{2}(s) f_{2}(s) f_{3}(s)\right] / 2 f_{2}(s) p(s)\right) d s+c_{3}$. Consider

$$
u_{35}=-\frac{2 a_{1}^{2} f_{0}(t)}{p^{2}(t) f_{2}(t)}+c_{2} \phi(t)[m s n(\xi) \pm i \operatorname{dn}(\xi)],
$$

where $\xi=c_{1} \varphi(t) x+\int\left(\left[-12 a_{1}^{2} f_{0}^{2}(s)+\left(2 m^{2}-1\right) p^{4}(s) f_{2}^{2}(s)+\right.\right.$ $\left.\left.2 p^{2}(s) f_{2}(s) f_{3}(s)\right] / 2 f_{2}(s) p(s)\right) d s+c_{3}$. Consider

$$
u_{36}=-\frac{2 a_{1}^{2} f_{0}(t)}{p^{2}(t) f_{2}(t)}+c_{2} \phi(t) \frac{\operatorname{sn}(\xi)}{1 \pm \operatorname{cn}(\xi)},
$$

where $\xi=c_{1} \varphi(t) x+\int\left(\left[-12 a_{1}^{2} f_{0}^{2}(s)+\left(2 m^{2}-1\right) p^{4}(s) f_{2}^{2}(s)+\right.\right.$ $\left.\left.2 p^{2}(s) f_{2}(s) f_{3}(s)\right] / 2 f_{2}(s) p(s)\right) d s+c_{3}$. Consider

$$
u_{37}=-\frac{2 a_{1}^{2} f_{0}(t)}{p^{2}(t) f_{2}(t)}+c_{2} \phi(t) \frac{\operatorname{cn}(\xi)}{\sqrt{1-m^{2}} \operatorname{sn}(\xi) \pm \operatorname{dn}(\xi)},
$$


where $\xi=c_{1} \varphi(t) x+\int\left(\left[-12 a_{1}^{2} f_{0}^{2}(s)+\left(2 m^{2}-1\right) p^{4}(s) f_{2}^{2}(s)+\right.\right.$ $\left.\left.2 p^{2}(s) f_{2}(s) f_{3}(s)\right] / 2 f_{2}(s) p(s)\right) d s+c_{3}$. Consider

$$
u_{38}=-\frac{2 a_{1}^{2} f_{0}(t)}{p^{2}(t)\left(m^{2}-1\right) f_{2}(t)}+c_{2} \phi(t) \frac{\operatorname{dn}(\xi)}{1 \pm m s n(\xi)},
$$

where $\xi=c_{1} \varphi(t) x+\int\left(\left[-12 a_{1}^{2} f_{0}^{2}(s)-\left(m^{4}-1\right) p^{4}(s) f_{2}^{2}(s)+\right.\right.$ $\left.\left.2\left(m^{2}-1\right) p^{2}(s) f_{2}(s) f_{3}(s)\right] / 2\left(m^{2}-1\right) f_{2}(s) p(s)\right) d s+c_{3}$. Consider

$$
\begin{aligned}
u_{39}= & -\frac{2 a_{1}^{2} f_{0}(t)}{p^{2}(t)\left(m^{2}-1\right) f_{2}(t)} \\
& +c_{2} \phi(t)[m s d(\xi) \pm \operatorname{nd}(\xi)],
\end{aligned}
$$

where $\xi=c_{1} \varphi(t) x+\int\left(\left[-12 a_{1}^{2} f_{0}^{2}(s)-\left(m^{4}-1\right) p^{4}(s) f_{2}^{2}(s)+\right.\right.$ $\left.\left.2\left(m^{2}-1\right) p^{2}(s) f_{2}(s) f_{3}(s)\right] / 2\left(m^{2}-1\right) f_{2}(s) p(s)\right) d s+c_{3}$. Consider

$$
u_{40}=\frac{2 a_{1}^{2} f_{0}(t)}{p^{2}(t)\left(m^{2}-1\right) f_{2}(t)}+c_{2} \phi(t) \frac{\mathrm{cn}(\xi)}{1 \pm m \operatorname{sn}(\xi)},
$$

where $\xi=c_{1} \varphi(t) x+\int\left(\left[-12 a_{1}^{2} f_{0}^{2}(s)+\left(m^{4}-1\right) p^{4}(s) f_{2}^{2}(s)+2(1-\right.\right.$ $\left.\left.\left.m^{2}\right) p^{2}(s) f_{2}(s) f_{3}(s)\right] / 2\left(1-m^{2}\right) f_{2}(s) p(s)\right) d s+c_{3}$. Consider

$$
u_{41}=\frac{2 a_{1}^{2} f_{0}(t)}{p^{2}(t)\left(m^{2}-1\right) f_{2}(t)}+c_{2} \phi(t)[\mathrm{nc}(\xi) \pm \mathrm{sc}(\xi)] \text {, }
$$

where $\xi=c_{1} \varphi(t) x+\int\left(\left[-12 a_{1}^{2} f_{0}^{2}(s)+\left(m^{4}-1\right) p^{4}(s) f_{2}^{2}(s)+2(1-\right.\right.$ $\left.\left.\left.m^{2}\right) p^{2}(s) f_{2}(s) f_{3}(s)\right] / 2\left(1-m^{2}\right) f_{2}(s) p(s)\right) d s+c_{3}$. Consider

$$
\begin{aligned}
u_{42}= & -\frac{2 a_{1}^{2} f_{0}(t)}{p^{2}(t)\left(m^{2}-1\right)^{2} f_{2}(t)} \\
& +c_{2} \phi(t) \frac{\operatorname{sn}(\xi)}{\operatorname{dn}(\xi) \pm \operatorname{cn}(\xi)},
\end{aligned}
$$

where $\xi=c_{1} \varphi(t) x+\int\left(\left[-12 a_{1}^{2} f_{0}^{2}(s)-\left(m^{2}+1\right)\left(m^{2}-\right.\right.\right.$ $\left.1)^{2} p^{4}(s) f_{2}^{2}(s)+2\left(m^{2}-1\right)^{2} p^{2}(s) f_{2}(s) f_{3}(s)\right] / 2\left(m^{2}-\right.$ $\left.1)^{2} f_{2}(s) p(s)\right) d s+c_{3}$. Consider

$$
u_{43}=-\frac{2 a_{1}^{2} f_{0}(t)}{p^{2}(t) m^{4} f_{2}(t)}+c_{2} \phi(t) \frac{\mathrm{cn}(\xi)}{\sqrt{1-m^{2}} \pm \operatorname{dn}(\xi)},
$$

where $\xi=c_{1} \varphi(t) x+\int\left(\left[-12 a_{1}^{2} f_{0}^{2}(s)-m^{4}\left(m^{2}-2\right) p^{4}(s) f_{2}^{2}(s)+\right.\right.$ $\left.\left.2 m^{4} p^{2}(s) f_{2}(s) f_{3}(s)\right] / 2 m^{4} f_{2}(s) p(s)\right) d s+c_{3}$.

Remark 2. There are some other hyperbolic function solutions and trigonometric function solutions which can be obtained from solutions (25)-(60) at the limit case when $m \rightarrow 1$ and $m \rightarrow 0$, but we omit them for simplicity.

Remark 3. By using our method, we can obtain many more general exact solutions including all the solutions given in [20] as special cases. More precisely, setting $h_{0}=h_{1}=h_{3}=0$, $h_{2}=1$, and $h_{4}=-1$, solution (18) is equivalent to (22) in [20]. Similarly, solution (20) is equivalent to (38) obtained in [20] setting $h_{0}=h_{4}=1, h_{1}=h_{3}=0$, and $h_{2}=-2$. Setting $h_{0}=h_{4}=1 / 4, h_{1}=h_{3}=0, h_{2}=-1 / 2$, and $m \rightarrow 1$, solution (52) is equivalent to (62) in [20]. The comparison between our method and the well known results in [20] shows that our method is more powerful than the method in [20] in constructing exact solutions.

\section{Conclusion}

With the help of symbolic computation, we employ the auxiliary equation method to find many new and more general exact solutions of the generalized KdV-MKdV equation with variable coefficients. This paper showed that the auxiliary equation method not only gives a unified formulation to construct a series of exact solutions but also provides a guideline to classify the types of solutions according to the given parameters. Applying the more generalized ansätz and auxiliary equation method to other variable-coefficients NPDEs is our future work.

\section{Conflict of Interests}

The authors declare that there is no conflict of interests regarding the publication of this paper.

\section{Acknowledgment}

This work is supported by the Hubei Provincial Department of Education (B2015146).

\section{References}

[1] R. Hirota, The Direct Method in Soliton Theory, Cambridge University Press, Cambridge, UK, 2004.

[2] M. J. Ablowitz and P. A. Clarkson, Solitons, Nonlinear Evolution Equations and Inverse Scattering, Cambridge University Press, New York, NY, USA, 1991.

[3] K. Konno and M. Wadati, "Simple derivation of Bäcklund transformation from Riccati form of inverse method," Progress of Theoretical Physics, vol. 53, no. 6, pp. 1652-1656, 1975.

[4] M. R. Miurs, Bäcklund Transformation, Springer, Berlin, Germany, 1978.

[5] M. Wang, X. Li, and J. Zhang, "Various exact solutions of nonlinear Schrödinger equation with two nonlinear terms," Chaos, Solitons \& Fractals, vol. 31, no. 3, pp. 594-601, 2007.

[6] X. Li and M. Wang, "A sub-ODE method for finding exact solutions of a generalized KDV-mKDV equation with highorder nonlinear terms," Physics Letters A, vol. 361, no. 1-2, pp. 115-118, 2007.

[7] D. Lu and C. Liu, "A sub-ODE method for generalized Gardner and BBM equation with nonlinear terms of any order," Applied Mathematics and Computation, vol. 217, no. 4, pp. 1404-1407, 2010.

[8] M. A. Abdou, "The extended F-expansion method and its application for a class of nonlinear evolution equations," Chaos, Solitons \& Fractals, vol. 31, no. 1, pp. 95-104, 2007.

[9] J.-L. Zhang, M.-L. Wang, Y.-M. Wang, and Z.-D. Fang, "The improved F-expansion method and its applications," Physics Letters, Section A: General, Atomic and Solid State Physics, vol. 350, no. 1-2, pp. 103-109, 2006.

[10] M. L. Wang and X. Z. Li, "Applications of F-expansion to periodic wave solutions for a new Hamiltonian amplitude equation," Chaos, Solitons and Fractals, vol. 24, no. 5, pp. 12571268, 2005.

[11] A.-M. Wazwaz, "Variants of the generalized KdV equation with compact and noncompact structures," Computers \& Mathematics with Applications, vol. 47, no. 4-5, pp. 583-591, 2004. 
[12] A.-M. Wazwaz, "A sine-cosine method for handling nonlinear wave equations," Mathematical and Computer Modelling, vol. 40, no. 5-6, pp. 499-508, 2004.

[13] W. Malfliet, "Solitary wave solutions of nonlinear wave equations," American Journal of Physics, vol. 60, no. 7, pp. 650-654, 1992.

[14] A. M. Wazwaz, "The tanh method for traveling wave solutions of nonlinear equations," Applied Mathematics and Computation, vol. 154, no. 3, pp. 713-723, 2004.

[15] J.-H. He and X.-H. Wu, "Exp-function method for nonlinear wave equations," Chaos, Solitons \& Fractals, vol. 30, no. 3, pp. 700-708, 2006.

[16] J.-H. He and M. A. Abdou, "New periodic solutions for nonlinear evolution equations using Exp-function method," Chaos, Solitons \& Fractals, vol. 34, no. 5, pp. 1421-1429, 2007.

[17] S. Liu, Z. Fu, S. Liu, and Q. Zhao, "Jacobi elliptic function expansion method and periodic wave solutions of nonlinear wave equations," Physics Letters A, vol. 289, no. 1-2, pp. 69-74, 2001.

[18] E. Fan and J. Zhang, "Applications of the Jacobi elliptic function method to special-type nonlinear equations," Physics Letters A, vol. 305, no. 6, pp. 383-392, 2002.

[19] X. Q. Zhao, H. Y. Zhi, and H. Q. Zhang, "Improved Jacobifunction method with symbolic computation to construct new double-periodic solutions for the generalized Ito system," Chaos, Solitons and Fractals, vol. 28, no. 1, pp. 112-126, 2006.

[20] H. Triki, T. R. Taha, and A.-M. Wazwaz, "Solitary wave solutions for a generalized KDV-mKDV equation with variable coefficients," Mathematics and Computers in Simulation, vol. 80, no. 9, pp. 1867-1873, 2010.

[21] C. Tian, "Symmetries and a hierarchy of the general KdV equation," Journal of Physics. A: Mathematical and General, vol. 20, no. 2, pp. 359-366, 1987.

[22] W. L. Chan and K.-S. Li, "Nonpropagating solitons of the variable coefficient and nonisospectral Korteweg-de Vries equation," Journal of Mathematical Physics, vol. 30, no. 11, pp. 25212526, 1989.

[23] J. F. Zhang, "Six sets of symmetries of the variable coefficient modified Korteweg-de Vries equation," Chinese Physics Letters, vol. 11, no. 1, pp. 4-7, 1994.

[24] Z. Fu, S. Liu, and S. Liu, "New kinds of solutions to Gardner equation," Chaos, Solitons and Fractals, vol. 20, no. 2, pp. 301309, 2004.

[25] D. Baldwin, Ü. Göktaş, W. Hereman, L. Hong, R. S. Martino, and J. C. Miller, "Symbolic computation of exact solutions expressible in hyperbolic and elliptic functions for nonlinear PDEs," Journal of Symbolic Computation, vol. 37, no. 6, pp. 669705, 2004.

[26] E. Yomba, "Construction of new soliton-like solutions for the $(2+1)$-dimensional Kadomtsev-Petviashvili equation," Chaos, Solitons and Fractals, vol. 22, no. 2, pp. 321-325, 2004.

[27] H. T. Chen and H. Q. Zhang, "New double periodic and multiple soliton solutions of the generalized $(2+1)$-dimensional Boussinesq equation," Chaos, Solitons and Fractals, vol. 20, no. 4, pp. 765-769, 2004.

[28] Y. Chen and Q. Wang, "A new general algebraic method with symbolic computation to construct new travelling wave solution for the (1+1)-dimensional dispersive long wave equation," Applied Mathematics and Computation, vol. 168, no. 2, pp. 11891204, 2005.
[29] D.-S. Wang and H.-B. Li, "Elliptic equation's new solutions and their applications to two nonlinear partial differential equations," Applied Mathematics and Computation, vol. 188, no. 1, pp. 762-771, 2007. 


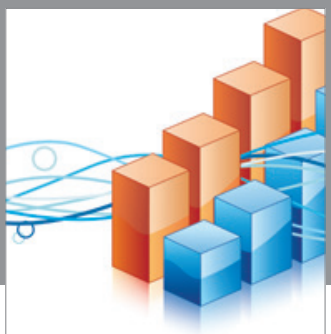

Advances in

Operations Research

vatem alat4

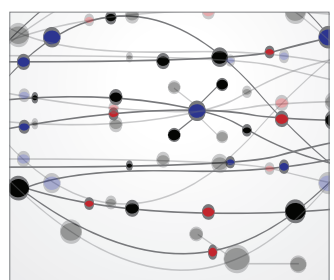

\section{The Scientific} World Journal
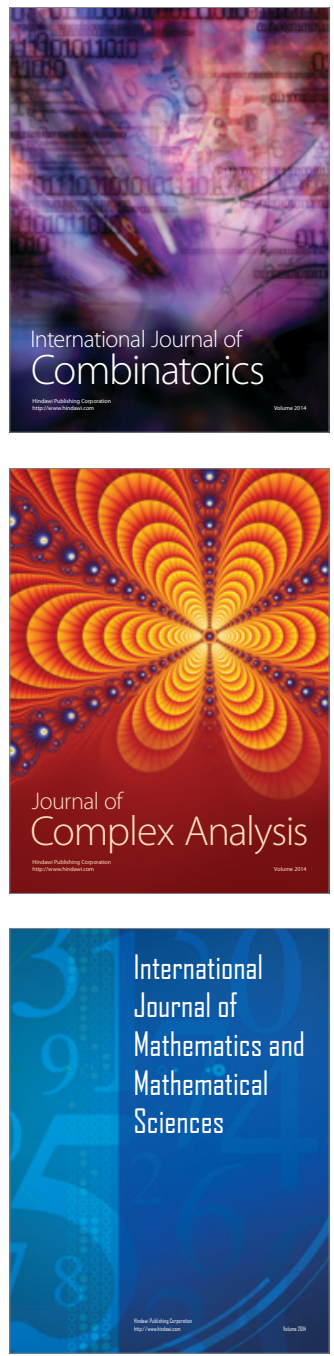
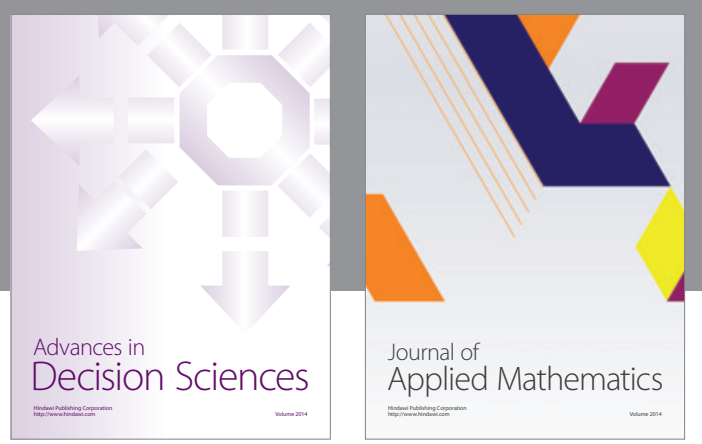

Algebra

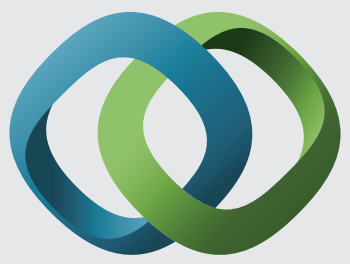

\section{Hindawi}

Submit your manuscripts at

http://www.hindawi.com
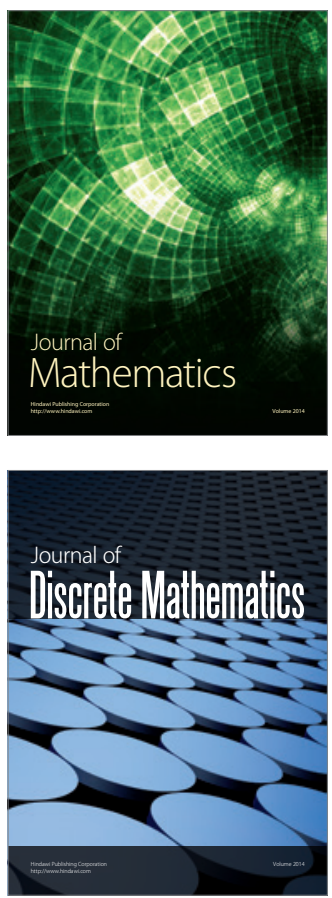

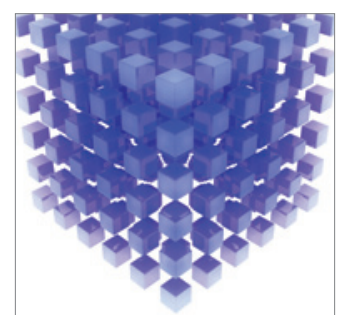

Mathematical Problems in Engineering
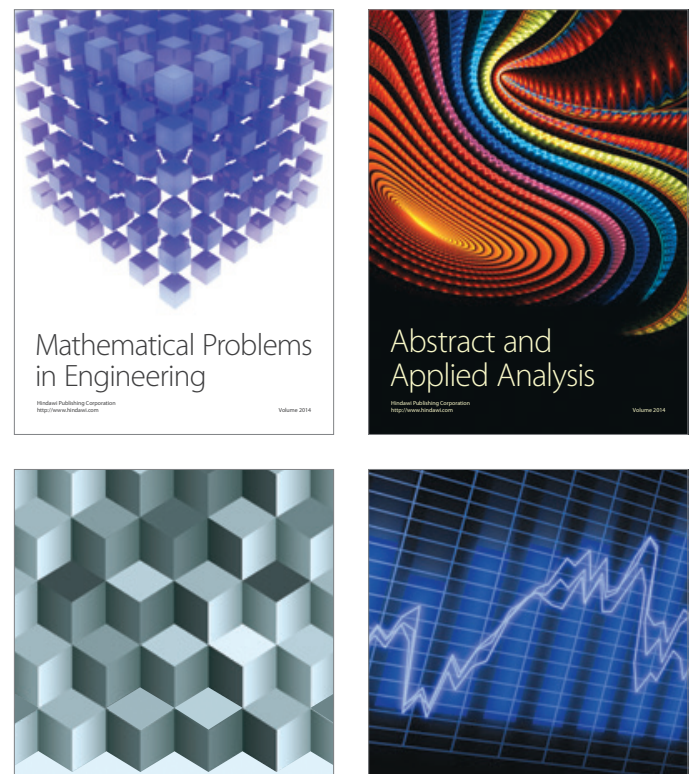

Journal of

Function Spaces

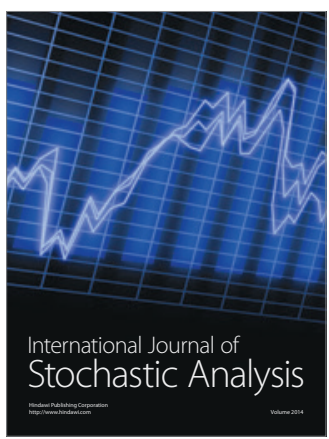

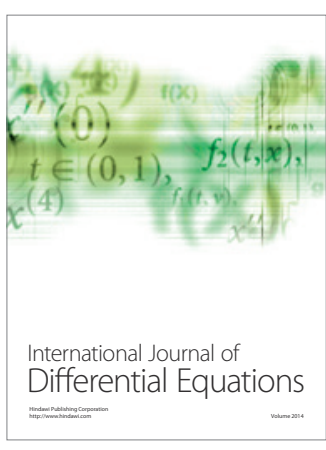
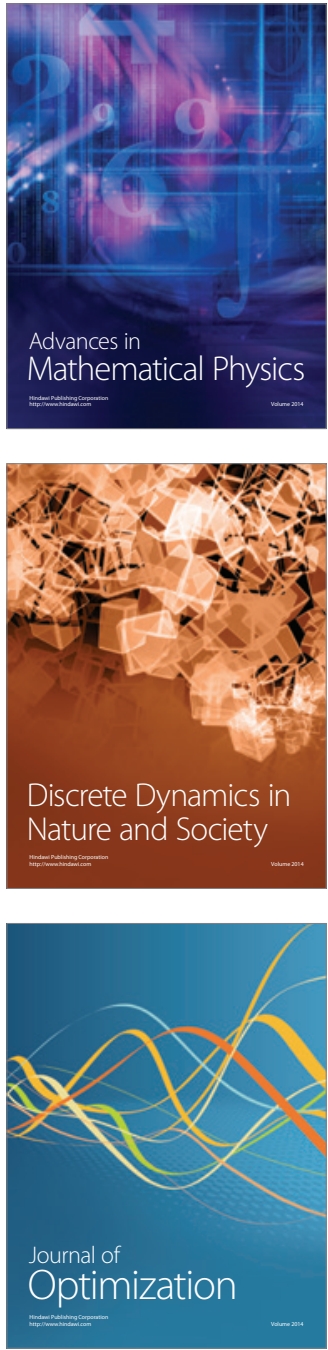